\title{
Feature Extraction Techniques for Iris Recognition System: A Survey
}

\author{
Adekunle, Y.A, Aiyeniko, O, Eze, M.O., Alao, O.D
}

\begin{abstract}
The extraction of features involves the method of converting the original pixel values of an image to more meaningful, useful and measurable information that can be used in other techniques, such as image processing, pattern recognition and machine learning. The feature extraction plays a predominant role in iris recognition in which also the recognition rate is determined. The effective recognition accuracy, reduction of misclassification of two iris templates mostly depends on feature extraction techniques. An efficient iris recognition system requires that the discriminating information presents in an iris pattern to be accurately obtained. This paper performed a literature review on different techniques of feature extraction of iris recognition. The recommendation was made on how these techniques can be further enhanced to produce an effective iris recognition system.
\end{abstract}

KEYWORDS- Biometrics, Computer vision, Feature extraction, Pattern recognition.

\section{INTRODUCTION}

The iris recognition is a biological verification technique that applies recognition of pattern approach through the resolution of the great images of the irises of a person's eyes. In recent times, the human iris has attracted much attention in biometrics [34]. Iris is a portion within the eye size of the pupil that is muscular in nature which controls the total light that goes into the eye [38]. The iris is so distinctive that is it impossible to have two irises being the same even for indistinguishable twins [19]. Iris has a particularly intriguing arrangement which produces abundant texture information. The special textural pattern presents in iris can be extracted using diverse techniques of feature extraction, and also can be saved as a template for

Manuscript received March 19, 2020

Adekunle, Y.A, Computer Science Department, Babcock University, Ilishan-Remo, Nigeria.

Aiyeniko, O, Computer Science Department, Babcock University, Ilishan-Remo, Nigeria (email: royalgrace2000@yahoo.com

Eze, M.O, Computer Science Department, Babcock University, Ilishan-Remo, Nigeria.

Alao, O.D, Computer Science Department, Babcock University, Ilishan-Remo, Nigeria. biometric in a repository for advance processing. The identified features produce local-global or both local and global information. Features aid in the analysis of the important textural characteristics of an iris [10].

The extraction of features from the portion of an iris is considered to be one of the significant steps in iris recognition system due to the fact that it is needed to be achieved with least possible classification and computational time [36]. The recognition rate of the iris recognition system answers to the number of features obtained from the iris portion [26]. A feature can be described as a function of one or more measurements of the object, which is computed such that it qualifies some significant characteristics of the object [3]. Only significant and discriminated information of iris texture must be extracted and encoded so that comparisons between templates can be made conveniently and correctly.

Robust recognition rate and a reduced classification time of two iris templates can be influenced by feature extraction technique [33].Development of an effective iris recognition system requires that best discriminating information available in an iris pattern to be properly extracted [3]. The complication in features extraction affects the complexity of the program and processing speed of the iris recognition system [26]. The relevant features of the iris must be encoded so that comparisons between templates can be made [11]. Inadequate iris images representation may affect the classification accuracy of the iris recognition system. It is very essential in iris recognition to look into the performance of techniques of feature extraction.

This paper conducted a general literature review on some commonly used feature extraction techniques in the iris recognition system. Suggestion was discussed on how these techniques can be improved to develop a better system of iris recognition.

\section{A. Feature Extraction Technique}

Feature extraction segregates many refined features from the iris template which can be referred to as feature vector. The feature vectors are further employed in matching the pattern [32]. Several feature extraction algorithms have been used in iris recognition [30].

\section{Gabor filter}

This is a linear-based filter used for the analysis of texture, which shows whether any specific frequency content in the image in certain directions in a localized region around the point or analysis region [43]. Gabor filters with unlike 
frequencies and orientations could be useful for the extraction of appropriate features from an image [1]. Two-dimensional Gabor filters have significant roles in image processing, particularly in feature extraction for analysis of texture, segmentation, analysis of documents, edge detection, image coding and image representation [40]. The technique renders resolution optimally in time domains, space and also produces a better visual image in the comprised texture images.

\section{B. Discrete Wavelet Transform (DWT)}

The DWT is an application of the wavelet transform using a discrete set of the wavelet scales and translations subsequent to distinct instructions [24]. Wavelets produce an orthonormal basis that is applied to decompose the signal processing and data compression. A signal is decomposed into a set of mutually orthogonal wavelet basis functions in wavelet analysis by DWT [21]. The operations differ from sinusoidal basis functions in that they are spatially localized that is, nonzero over only part of the total signal length.

\section{Discrete Cosine Transform (DCT)}

The technique states a set sequence of data points based on the addition of cosine functions oscillating at diverse frequencies [27]. When compared with the existing transforms, it also attempts to de-correlate a given signal [20]. DCT has been widely applied in signal or image processing particularly for lossy compression due to its excellent energy compaction to predict according to its local distinctiveness [24].

\section{Haar Wavelet Transform (HWT)}

This feature extraction method applies a sampling approach in which rows of the transformation matrix act as a sample of finer and finer resolutions, this is a great and pedagogical method of feature extraction [17]. HWT uses a pair of low-pass and high-pass filters to image decomposition first in image colours and then image rows independently. HWT works on features by the computation of the additions and differences of adjacent elements, it functions first on adjacent elements horizontally and then on adjacent elements vertically [4]. First applies in image column then in image rows one by one. HWT consists of four sub-bands, these are LL1, HL1, LH1 and HH1 up to four levels of decomposition are achieved to obtain the detailed image.

\section{E. Local Binary Pattern (LBP)}

It is a local texture operator that does respond to gray-scale changes [22]. LBP is obtained from a common description of texture in a local neighbourhood [35]. The LBP operator thresholds is a neighbourhood gray value centre, which shows the output as a binary code that defines the local texture pattern. LBP performs very well with areas demanding fast feature extraction and texture classification due to its discriminative power and computational simplicity [28]. The technique has become a widely used approach in different fields like iris recognition, visual inspection, image retrieval, remote sensing, face recognition, environmental modelling and outdoor scene analysis.

\section{F. Principal Component Analysis (PCA)}

The PCA represents the classical method for compressing huge data dimensionality to reduced dimensional ones for data analysis, visualization and feature extraction [37]. It uses a procedure mathematically that transforms a number of correlated variables into a smaller number of uncorrelated variables, which is called a principal component which can be used to reconstruct all of the information within a dataset and can be tested to which level attest image couples with an image of a training set. PCA compresses larger correlated variables to a reduced number of uncorrelated variables [19]. The reduced uncorrelated variables are known as principal components. These components generally denote the maximum variance available and thus showing the internal structure of the data.

\section{RELATED WORK}

Zhao and Kumar [42] proposed a system for iris detection using deep learning-based unified and generalizable framework. The system used the state of art with iris-specific Mask R-CNN, which adequately performed better reliable iris detection. Primary segmentation locating iris/non-iris pixels, an optimized convolutional network was adopted, which produced spatially corresponding iris feature descriptors. An Extended Triplet Loss (ETL) function was designed specially to combine the bit-shifting and non-iris masking, which are important for learning meaningful and discriminative spatial iris features. Experimental analysis on four publicly available datasets showed that the framework consistently outperformed several classic and state-of-the-art iris recognition systems. Abikoye et al. [2] focused on determining the performance of some methods of extraction for a feature by conducting an analysis comparatively on these methods: GWT, SIFT and Haar Wavelet Transform. The system was tested using CASIA iris dataset. Experimental analysis of each feature extraction technique gave different results. GWT technique recorded the best performance out the methods based on training time, testing time and recognition rate.

Biu et al. [8] improved recognition system by generating a binary bit sequence of iris. The information produced can be employed to determine different ailments of the eye. A predefined image was applied to obtain an iris by scanning for 8 different stages. Decomposition of packet wavelet was applied to produce 64 wavelet packages bit iris code. The evaluation of the system was done using the UBIRIS v.1 database. The classification of the iris codes was achieved with Hamming distance. The experimental result gave $98 \%$ of True Acceptance Rate and 1\% of False Rejection Rate.

Biu et al. [8] enhanced the authentication process. A binary bit sequence of the iris was generated, which contained several vital information that was used to calculate the mean energy and maximum energy that went into the eye with an adopted threshold value. The information generated can further be used to find out different eye ailments. An iris was obtained using a predefined iris image which was scanned through (8) different stages and wavelet packet decomposition was used to generate 64 wavelet packages bit iris code so as to match 
the iris codes with Hamming distance criteria and evaluate different energy values. The system was evaluated using the UBIRIS v.1 Database. The experimental results showed 98\% True Acceptance Rate and 1\% False Rejection Rate and this was because some of the irises weren't properly captured during the iris acquisition phase.

Ranjan et al. [42] examined the pattern of the iris in an eye. When capturing the iris under not as much as perfect condition antiquities in image occur such as diverse type of noise and reflections from light sources, artefacts that introduce error in the iris recognition and also affect the execution. The value of images as data from a dataset obtained using a standard camera was reviewed, the most vital areas identified, and the complete general recognition effectiveness was considered.

Solanke et al. [39] mentioned the enhancement of normalization process based on Daugman's rubber sheet model and feature extraction was based cumulative sum based change analysis. Iris features were extracted and the iris template was generated by horizontally and vertically grouping the iris texture features as iris codes. The localization and segmentation based on black hole search method effectively separated the region of interest from the input eye image. The normalization method and feature extraction methods were quite less time consuming and produced the desired results.

Bansal et al. [31] employed the extraction of feature technique based on a statistical approach using the correlation between adjacent pixels. The classification was achieved using hamming distance. The performance evaluation of the system was done at the diverse threshold in the distance metrics. Experimental results with several statistical characteristics of iris images revealed a major enhancement in ERR when there was an increase in statistical parameters for feature extraction.

Devi et al. [23] developed a system using compound local binary pattern approach. The abundant distinctive features were obtained using Compound Local Binary Pattern (CLBP). Artificial Neural Network was applied to classify the operators. Evaluation of the system with fifty images of the eye was done using the CASIA database. The proposed system with Neural Network produced an improved accuracy of $96 \%$ when compared with the existing feature extraction methods.

Kumar et al. [26] presented an iris feature extraction and recognition based on $2 \mathrm{D}$ discrete cosine transform. The iris localization was done by a circular Hough transform. After locating the iris, normalization of iris images was achieved by Baughman rubber-sheet model so as to transform the iris region into a fixed dimension. 2D DCT was used to extract the most discriminating features of iris. The feature extraction capabilities of DCT were tested on two publicly available CASIA V4 and IIITD database. The hamming distance was used for matching the iris templates. For verification, a variable threshold value was applied to the distance metric and false acceptance rate and false rejection rate were recorded. An accuracy of $99.4 \%$ and $98.4 \%$ were recorded for the two datasets.
Harsha et al.[37] described a distinct method for recognition using iris. The study applied the canny edge detection and a circular Hough transform to find the boundaries of iris in the eye. Discrete wavelet transform of two-level was used to obtain the patterns in an individual's eye. Recognition was achieved by distance measure classifier. The system gave a better total successive rate (TSR) with a reduction in EER, FAR and FRR.

Kerim et al. [25] applied dual techniques for extraction of iris feature from normalized iris image by using statistical measurements, the first approach used first-order statistics and the second approach employed second-order statistics. The system was aimed to obtain feature correctly from pure iris region and isolate the eyelid and eyelash which are covers some parts of the iris region. The proposed methods used weights within pattern matching measures depending on purity iris region. The proposed methods have been tested by using the iris data set (CASIA v4.0-interval), and (CASIA v1.0- interval) iris image database. Experimental results showed that the first proposed method has $99.4 \%$ accuracy rate with (CASIA v4.0- interval) and $98.5 \%$ with (CASIA v1.0- interval), the second proposed method has $86.67 \%$ with (CASIA v4.0- interval).

Gandhi et al. [13] developed iris recognition by using improved SIFT for extraction of the feature. The technique was used to describe the local properties on an image by extracting the image from a collection of key points, the keypoints invariant to lighting condition, rotation and scale changes are detected first, the feature vector for each keypoint was produced through the gradient directions of pixels in a block centred point. The study concluded that the SIFT algorithm made preprocessing easier and can be used although some issues need to be considered before it can be widely implemented.

Oluwakemi et al. [29] applied a new algorithm for iris feature extraction. The feature extraction was facilitated while the localized iris was transformed from a cartesian coordinate system to a polar coordinate system. The algorithm used Fast Wavelet Transform to extract features of iris. This algorithm is fast with lower complexity rate.

Patil et al. [31] conducted a review on feature extraction techniques for iris recognition system. Three feature extraction methods were considered; Haar Wavelets, circular symmetric filter, Lifting wavelet transform for performance analysis of iris recognition. The experimental results gave percentage accuracies; $99.85 \%$ of circular symmetric filter, $98.78 \%$ of lifting wavelet transform and $95 \%$ of Haar wavelets.

Chirchi et al. [9] designed a system for iris recognition using feature extraction based technique of five-level decomposition by application of $\mathrm{db} 2$ and $\mathrm{db} 4$ and Haar wavelet. The system recorded a recognition rate of $99.97 \%$ with its speed faster and equal error rate smaller than existing systems.

He, Feng et al. [15] proposed a novel iris feature extraction system based on local binary pattern (LBP) images and the chunked encoding method. LBP was employed to normalize the iris image and obtain the iris' LBP image. The iris's feature was extracted through the chunked 
encoding method based on the iris' statistical information. Iris recognition and classification was done using Hamming distance. Experimental results showed that this algorithm can get a higher recognition rate than the existing iris feature extraction method, which demonstrated the efficiency of the proposed method.

\section{SUMMARY AND DISCUSSION}

The critical phase of the iris recognition system remains the feature extraction. The technique of feature extraction entails the method of reducing the number of features needed to describe huge information available in an iris pattern. The decrease in recognition time and the recognition rate of two iris templates are subject to efficient feature extraction techniques. Researchers in the past have employed diverse techniques to improve the level of accuracy. A combination (fusion) of homogenous or heterogeneous features of iris image such as Gabor filters (1-D Gabor filter, 2-D Gabor filter), Local Binary Pattern (Local Ternary Pattern) and its variants have been employed to develop a better system of iris recognition. In spite of all the improvements recorded so far, there is still a challenge of how to ascertain the relevancy of the features to be used for classification. There is a need to apply feature selection approach on extracted features before the recognition phase.

Several feature selection techniques have been employed such as Principal Component Analysis (PCA), Linear Discriminant Analysis (LDA) and Independent Component Analysis (ICA) in the pattern recognition, image processing and computer vision [9]. These techniques have not really produced the most relevant or optimized features. It is, therefore, necessary to introduce metaheuristic optimization methods for feature selection. With the feature selection, the complexity and computational cost of the classifier can be reduced by minimizing the number of features to be used into measurable forms while still maintaining acceptable recognition accuracy [27][16]. Obtaining an optimal feature subset in feature selection turns out to be usually intractable [41], and many other problems related to feature selection shown to be nondeterministic polynomial hard problem (NP) ][18]. A problem like this requires not just the conventional optimization techniques, but a robust optimization approach such as metaheuristic optimization methods.

The introduction of metaheuristic optimization algorithms such as firefly optimization, cuckoo optimization, artificial bee colony optimization, ant colony optimization, gravitational search optimization and bacterial optimization, which are largely nature-inspired computational optimization techniques can be applied in future work to select the most relevant and discriminant features before fusion phase. This will contribute to the reduction of irrelevant features at the classification stage, thus also minimize the level of misclassification in the iris recognition system.

\section{CONCLUSION}

Feature extraction is a dimensionality reduction method which involves the transformation of original (raw) features into reduced or manageable features which contain the most discriminatory information. This paper carried a survey on some different feature extraction techniques for iris recognition system. Based on the survey of iris feature extraction techniques, it was suggested that enhancement should be considered on iris recognition by application of metaheuristic optimization algorithms to select the most relevant features from iris extracted features which will improve the iris recognition performance. The review of the feature extraction techniques provided a platform for the development of a robust iris recognition system in future work.

\section{REFERENCES}

[1] Abhishree, T. M., Latha, J., Manikantan, K., \& Ramachandran, S. (2015). Face Recognition using Gabor filter based Feature Extraction with Anisotropic Diffusion as a pre-processing technique. Procedia Computer Science, 45(C),

312-321. https://doi.org/10.1016/j.procs.2015.03.149

[2] Abikoye, O. C., Aro, T. O., Ogundokun, O., \& Akande, H. B. (2019). Comparative Analysis of Selected Feature Extraction Techniques for Iris Recognition System. FUW Trends in Science \& Technology Journal, 3(2), 541-545.

[3] Adegoke, B. O, Omidiora, E. O, Ojo, J. A., \& Falohun, S. A. (2013). Iris feature extraction: A survey. Computer Engineering and Intelligent, 4(9), 7-14.

[4] Arjunan, T. (2015). Iris Recognition using Invariant Moment Features Based on Haar Wavelet Decomposition. Indian Journal of Engineering, 12(30), 54-59.

[5] Ashwini, M. B., Mohammad, I. \&, \& Fawaz, A. (2015). Evaluation of Iris Recognition System on Multiple Feature Extraction Algorithms and its Combinations. International Journal of Computer Applications Technology and Research, 4(8), 592-598. https://doi.org/10.7753/IJCATR0408.1002

[6] Bansal, A., Agarwal, R., \& Sharma, R. K. (2016). Statistical feature extraction based iris recognition system. [7] Sadhana, 41(5), 507-518. https://doi.org/10.1007/s12046-016-0492-9

[8] Biu., A. H., Husain, R., \& Magaji, A. S. (2018). An Enhanced Iris Recognition and Authentication System Using Energy Measure. Science World Journal, 13(1), 11-17.

[9] Chirchi, V. R. E., \& Waghmare, L. M. (2013). Feature Extraction and Pupil Detection Algorithm Used for Iris Biometric Authentication System. 6(6), 141-160. https://doi.org/10.14257/ijsip.2013.6.6.14

[10] Chouhan, B., \& Shukla, S. (2010). Analysis of statistical feature extraction for Iris Recognition System using Laplacian of Gaussian filter. International Journal of Applied Engineering Research, Dindigul, 1(3), 528-535.

[11] Devi, K., Gupta, P., Grover, D., \& Dhindsa, A. (2016). An Effective Feature Extraction Approach for Iris Recognition System. Indian Journal of Science and Technology, 9(December), 1-5. https://doi.org/10.17485/ijst/2016/v9i47/106827 
[12] Fauna, K. K., \& Athira, P. \& R. K. J. S. (2016). A Review on Iris Feature Extraction Methods. International Journal of Engineering Research and General Science, $4(2), 663-667$.

[13] Gandhi, K. M., \& Kulkarni, P. R. H. (2014). Sift Algorithm for Iris Feature Extraction. Global Journal of Computer Science and Technology Graphics \& Vision, 14(3), 0-6.

[14] Harsha, R., \& Ramesha, K. (2015). DWT Based Feature Extraction for Iris Recognition. International Journal of Advanced Research in Computer and Communication Engineering, 4(5), 300-306. https://doi.org/10.17148/IJARCCE.2015.4567

[15] He, Y., Feng, G., Hou, Y., Li, L., \& Micheli-Tzanakou, E. (2011). Iris feature extraction method based on LBP and chunked encoding. Proceedings - 2011 7th International Conference on Natural Computation, ICNC 2011, 3, 1663-1667. https://doi.org/10.1109/ICNC.2011.6022302

[16] Hira, Z. M., \& Gillies, D. F. (2015). A Review of Feature Selection and Feature Extraction Methods Applied on Microarray Data. Advances in Bioinformatics, 198-363. https://doi.org/10.1155/2015/198363

[17] Ibrahim, A. A. (2014). Iris Recognition using Haar Wavelet Transform. Journal of AL-Nahrain-University Science, 17(1), 80-86.

[18] Imani, M. B., Pourhabibi, T., Keyvanpour, M. R., \& Azmi, R. (2012). A New Feature Selection Method Based on Ant Colony and Genetic Algorithm on Persian Font Recognition. International Journal of Machine Learning and Computing, 2(3), 278-282.

[19] Jain, A. K., Ross, A., \& Pankanti, S. (2006). Biometrics: A Tool for Information Security. IEEE Transactions on Information Forensics and Security, 1(2), 125-143. https://doi.org/10.1109/TIFS.2006.873653

[20] Joshua, T. P., Arrivukannamma, M., \& Sathiaseelan, J. G. R. (2016). Comparison of DCT and DWT Image Compression. International Journal of Computer Science and Mobile Computer, 5(4), 3-8.

[21] Jyoti, P., Parvati, B., \& Kumar, R., \& Agrawal, S. L. (2015). Performance Review of IRIS Recognition Systems. International Journal of Computer Systems, 02(12), 564-566.

[22] Kapil, D., \& Jain, E. A. (2015). A Brief Review on Feature-Based Approaches for Face Recognition. International Journal of Science and Research, 4(5), 273-277.

[23] Kaur, M. \& Jasjit, K. (2017). Review of Face Recognition Techniques. International Journal of Computer Applications, 164(6), 31-35.

[24] Kaur, R., \& Choudhary, P. (2016). A Review of Image Compression Techniques. International Journal of Computer Applications, 142(1), 8-11.

[25] Kerim, A. A \& Mohammed, S. J. (2014). New Iris Feature Extraction and Pattern Matching Based on Statistical Measurement. 3(5), 226-231.
[26] Kumar, A., Potnis, A., \& Singh, A. P. (2016). Iris recognition and feature extraction in iris recognition System by employing 2D DCT. International Research Journal of Engineering and Technology, 3(12), 503-510.

[27] Miche, Y., Bas, P., Lendasse, A., Jutten, C., \& Simula, O. (2007). Advantages of Using Feature Selection Techniques on Steganalysis Schemes. 9th International Work-Conference on Artificial Neural Networks, IWANN'2007, 606-613. https://doi.org/10.1007/978-3-540-73007-1_73

[28] O’Connor, B., Roy, K., Shelton, J., \& Dozier, G. (2014). Iris Recognition Using Fuzzy Level Set and GEFE. International Journal of Machine Learning and Computing, 4(3), 225-231.

https://doi.org/10.7763/IJMLC.2014.V4.416

[29] Oluwakemi, A., Sadiku, J. S., Kayode, A., \& Rasheed, J. (2014). Iris Feature Extraction for Personal Identification using Fast Wavelet Transform (FWT). International Journal of Applied Information Systems, 6(9), 1-6.

[30] Patel, K. S., Pithadiya, K. J., \& Chauhan, J. C. (2015). International Journal of Advanced Engineering and Research 2D Palmprint Recognition: A Survey. International Journal of Advanced Engineering and Research Development, 2(7), 332-337.

[31] Patil, R. B., \& Deshmukh, R. R. (2013). A Review on Feature Extraction Techniques of Iris. International Journal of Engineering Research \& Technology, 2(12), 2909-2912.

[32] Prajwala, N. B., \& Pushpa, N. B. (2019). Matching of Iris Pattern Using Image Processing. International Journal of Recent Technology and Engineering, 8(2), 21-23. https://doi.org/10.35940/ijrte.B1004.0982S1119

[33] Rampally, D. (2010). Iris Recognition Based on Featured Extraction. Manhattan.

[34] Ranjan, S. Prabu, S., Swarnalatha, P., Magesh, G., \& Sundararajan, R. (2017). Iris Recognition System. International Research Journal of Engineering and Technology, 04(12), 864-868. https://doi.org/10.14569/ijacsa.2010.010106

[35] Sarode, N. S., \& Patil, A. M. (2015). Iris Recognition using LBP with Classifiers-KNN and NB. International Journal of Science and Research, 4(1), 1904-1908.

[36] Shah, J. H., Sharif, M., Raza, M., \& Azeem, A. (2013). A Survey: Linear and Nonlinear PCA Based Face Recognition Techniques. International Arab Journal of Information Technology, 10(6), 536-545.

[37] Shi, J.-X., \& Gu, X.-F. (2010). The comparison of iris recognition using principal component analysis, independent component analysis and Gabor wavelets. Computer Science and Information Technology (ICCSIT), 2010 3rd IEEE International Conference on, 1(1), 61-64. https://doi.org/10.1109/ICCSIT.2010.5563947

[38] Shirke, S. D., \& Gupta, D. (2013). Iris Recognition Using Gabor. International Journal of Computer Technology \& Application, 4(1), 1-7. 
[39] Solanke, S. B., \& Deshmukh, R. R. (2017). Enhanced Feature Extraction Technique for Iris Template Generation. International Journal of Computer Technology \& Application, 8(August), 499-506.

[40] Thiyaneswaran, B., \& Padma, S. (2015). Analysis of Gabor Filter Parameter for Iris Feature Extraction. International Journal of Advanced Computer Technology, 3(5), 45-48.

[41] Yu, L., \& Liu, H. (2004). Efficient Feature Selection via Analysis of Relevance and Redundancy. Journal of Machine Learning Research, 5(1), 1205-1224. https://doi.org/10.1145/1014052.1014149

[42] Zhao, Z., \& Kumar, A. (2019). A deep learning based unified framework to detect, segment and recognize irises using spatially corresponding features. Pattern Recognition, 93, 546-557. https://doi.org/10.1016/j.patcog.2019.04.010

[43] Zhen, X. (2013). Feature Extraction and Representation for Human Action Recognition. Retrieved from

http://www.intechopen.com/books/face-recognition/featur e-extraction-and-representation-for-recognition 